\title{
Editorial
}

\section{Giuseppe Lippi}

\section{Sepsis biomarkers: past, present and future}

https://doi.org/10.1515/cclm-2018-1347

According to the Third International Consensus Definitions for Sepsis and Septic Shock [1], sepsis is currently defined as severe organ dysfunction caused by a dysregulated host response to infection associated with severe injuries to tissues and organs, up to septic shock, multiorgan failure (MOF) and death. Sepsis is a major public healthcare issue, being now considered as the leading cause of mortality from infections, accounting for more than 20 billion US $\$$ hospital costs each year in the US [1].

Recent statistics shows that the actual prevalence of sepsis is as high as $6 \%$ in hospitalized adults [2], whilst reliable predictions suggest that its future incidence may nearly double in the next 30 years [3]. The cumulative inhospital mortality is approximately $15 \%$ [2], whereas a much higher death rate is observed in intensive care units (ICUs), typically comprised between $20 \%$ and $45 \%$ [4]. Although both Gram-positive and -negative bacteria may trigger sepsis, the most frequent of which are Staphylococcus aureus (20.5\% of cases), Pseudomonas species (19.9\%), Escherichia coli (16.0\%), Klebsiella species (12.7\%), Enterococcus (10.9\%) and Staphylococcus epidermidis (10.8\%), sepsis can also be frequently develop in patients infected by Candida (17.0\%) and other microorganisms [5]. The lungs $(36 \%-42 \%)$ are the more frequent infection sites in patients with severe sepsis, followed by genitourinary tract $(10 \%-18 \%)$, abdomen $(8 \%-9 \%)$ and wounds or soft tissues $(7 \%-9 \%)$, whilst no precise source of bacteremia can be identified is as many as $20 \%$ of patients [5].

Irrespective of the fact that a timely and accurate diagnosis of sepsis is essential to reverse its otherwise unfavorable clinical course, the diagnostic approach remains challenging. Some scoring systems have been developed in recent years, including the host systemic inflammatory response syndrome (SIRS) criteria, the sequential [sepsis-related] organ failure assessment (SOFA) and the quick SOFA (qSOFA) scores [6]. Nevertheless, all these tools are primarily intended for predicting outcomes, especially death, whilst their early diagnostic efficiency remains unsatisfactory [7]. Even if the Sepsis-3 Task Force reiterates the concept that sepsis shall be identified using clinical criteria for life-threatening organ dysfunction and blood culture [1], both these approaches would only be efficient for delayed diagnosis and thus partially unable to substantially ameliorate an unfavorable prognosis.

For a long time, standard blood culture techniques have represented the only reliable means for diagnosing sepsis, and are still regarded as the gold standard reference methods for detecting and isolating pathogenic organisms from sterile body fluid specimens [8]. Unfortunately, however, blood culture has many drawbacks such as a long turnaround time (TAT) (i.e. $6 \mathrm{~h}$ to 5 days are needed for microorganisms to grow to detectable levels, with an additional 24-48 h needed for testing antibiotic susceptibility), low sensitivity, large sample volume, frequent need for repeated testing, risk of false negative test results after initiation of antibiotic therapy (between 30\% and $63 \%$ of cases) and preanalytical variables (e.g. $2 \%-4 \%$ sample contamination due to non-observance of standard antiseptic procedures during blood drawing) (Figure 1). Finally, albeit that automated approaches have been developed [9], blood culture remains a labor-intensive and timeconsuming procedure in many clinical laboratories.

Due to these inherent limitations, a large armamentarium of serum (or plasma) sepsis biomarkers has been commercialized over the past decades. These typically include C-reactive protein (CRP), procalcitonin, presepsin, interleukin 6 (IL-6), lipopolysaccharide-binding protein (LBP), neutrophil CD64 (nCD64), soluble triggering receptor expressed on myeloid cells-1 (sTREM-1) and the serum soluble urokinase-type plasminogen activator receptor (suPAR), among others. Although none of these biomarkers would thoughtfully fulfil all the ideal features of a sepsis biomarker, as briefly summarized in Table 1 , stronger clinical evidence of clinical usefulness has emerged for procalcitonin, presepsin and CRP from many published studies and meta-analyses. Both procalcitonin and presepsin now appear to be the most promising tests, not only for early diagnosis of sepsis, but also for garnering valuable prognostic information and for guiding therapeutic decision-making (i.e. antibiotic stewardship) [7, 10-11]. Nonetheless, their measurement is also plagued by some important drawbacks, such as insufficient standardization [12], the inability to provide information on causative microorganism and suboptimal diagnostic accuracy. Regarding this last aspect, a recent study published by Brodska et al. showed that the diagnostic 


\begin{tabular}{|c|c|c|c|}
\hline Diagnostic features & Blood culture & Serum biomarkers & Molecular biology \\
\hline Etiological diagnosis & Yes & No & Yes \\
\hline Standardization & Acceptable & Limited & Limited \\
\hline Rapidity & Limited & High & Limited \\
\hline Accuracy & Partial & Partial & Partial \\
\hline Predictiveness & No & Yes & No \\
\hline Antibiotic resistance & Yes & No & No \\
\hline Preanalytical issues & Yes & Limited & Yes \\
\hline
\end{tabular}

Figure 1: Diagnostic features of past, present and future sepsis biomarkers.

Table 1: Ideal features of sepsis biomarkers.

- Being present at symptoms onset (or even earlier), to allow for an early diagnosis

- Being highly sensitive and specific for infections, to allow for an accurate differential diagnosis between infectious and non-infectious diseases

- Being capable of identifying the causative microorganism

- Being informative on the clinical course

- Providing valuable information on the prognosis

- Guiding therapeutic decisions (e.g. antibiotic stewardship)

accuracy of some of the currently available biomarkers remains limited (i.e. $<80 \%$ ), and that presepsin even failed to outstrip more conventional sepsis biomarkers such as procalcitonin and CRP for both diagnosing sepsis and prognosticating death in critically ill patients [13].

Nucleic acid amplification is one of the most promising perspectives in sepsis diagnostics. The current techniques are essentially based on rapid amplification of DNA or RNA of pathogen origin, up to obtaining detectable levels which can then be assayed using mass spectrometry (MS), highresolution melting or sequencing. Several commercial methods have been commercialized and cleared by many regulatory agencies worldwide, as is thoughtfully reviewed elsewhere [8]. Albeit recent evidence suggests that the diagnostic performance of these assays is comprised between $70 \%$ and $90 \%$, the ability to accurately detect pathogens is still limited by some preanalytical issues, the need for effective lysis across a broad range of bacteria, interference from host DNA or other inhibitory substances, off-target interactions and amplification bias [8]. Moreover, antimicrobial stewardship may remain virtually unchanged even after molecular biology detection, so that clinical efficiency and cost-effectiveness of these techniques remain largely untested [14]. Hence, further refinements of molecular assays would be needed to overcome the current limitations in their diagnostic performance.

Early diagnosis of sepsis remains crucial for the managed care of patients with severe infections, who need timely treatment much earlier than after any signs and symptoms of organ failure have appeared. Prompt pathogen identification would also be highly effective for limiting inappropriate antibiotics usage, thus lowering the risk of antimicrobial resistance, which is one of the biggest threats to global health according to the World Health Organization (WHO). As previous work suggested, neither the past, present or future tests would fulfil all the features of an ideal sepsis biomarker and thus being considered the panacea that clinicians are expecting (Table 1). On the other hand, the integration of these existing technologies would probably be effective for counterweighing individual drawbacks but will exponentially increase the healthcare expenditure. Future costeffectiveness studies aimed at comparing and validating their diagnostic usage, alone or in combination, will thus be necessary.

The most reliable strategy for early diagnosis of sepsis appears to be that suggested by Schuetz et al. in this issue of the journal [15], relying on diagnostic algorithms integrating the pretest probability of infection, clinical features and results of in vitro diagnostic testing (e.g. procalcitonin). This approach, entailing close collaboration and cooperation between laboratory professionals and clinicians, reflects the current value of the 
so-called "clinical laboratory stewardship" [16], which must be seen as an essential step forward for improving the appropriateness of laboratory test ordering and the accuracy of interpretation of test results. Nonetheless, additional practical issues should be addressed, such as the current lack of standardization of procalcitonin immunoassays, as is also highlighted in another interesting article published by Chambliss et al. in this issue of the journal [17].

Author contributions: The author has accepted responsibility for the entire content of this submitted manuscript and approved submission.

Research funding: None declared.

Employment or leadership: None declared.

Honorarium: None declared.

\section{References}

1. Singer M, Deutschman CS, Seymour CW, Shankar-Hari M, Annane $\mathrm{D}$, Bauer $\mathrm{M}$, et al. The third international consensus definitions for sepsis and septic shock (Sepsis-3). J Am Med Assoc 2016;315:801-10.

2. Rhee C, Dantes R, Epstein L, Murphy D, Seymour CW, Iwashyna TJ, et al. Incidence and trends of sepsis in US hospitals using clinical vs claims data, 2009-2014. J Am Med Assoc 2017;318:1241-9.

3. Angus DC, Kelley MA, Schmitz RJ, White A, Popovich J Jr. Caring for the critically ill patient. Current and projected workforce requirements for care of the critically ill and patients with pulmonary disease: can we meet the requirements of an aging population? J Am Med Assoc 2000;284:2762-70.

4. Melville J, Ranjan S, Morgan P. ICU mortality rates in patients with sepsis compared with patients without sepsis. Crit Care 2015;19(Suppl 1):14.

5. Mayr FB, Yende S, Angus DC. Epidemiology of severe sepsis. Virulence 2014;5:4-11.

6. Marik PE, Taeb AM. SIRS, qSOFA and new sepsis definition. J Thorac Dis 2017;9:943-5.

7. Lippi G, Montagnana M, Balboni F, Bellone A, Casagranda I, Cavazza M, et al. Academy of Emergency Medicine and Care-Society of Clinical Biochemistry and Clinical Molecular
Biology consensus recommendations for clinical use of sepsis biomarkers in the emergency department. Emerg Care J 2017;13:6877.

8. Sinha M, Jupe J, Mack H, Coleman TP, Lawrence SM, Fraley SI. Emerging technologies for molecular diagnosis of sepsis. Clin Microbiol Rev 2018;31. doi: 10.1128/CMR.00089-17. Print 2018 Apr.

9. Da Rin G, Zoppelletto M, Lippi G. Integration of diagnostic microbiology in a model of total laboratory automation. Lab Med 2016;47:73-82.

10. Yang HS, Hur M, Yi A, Kim H, Lee S, Kim SN. Prognostic value of presepsin in adult patients with sepsis: systematic review and meta-analysis. PLoS One 2018;13:e0191486.

11. Bartoletti M, Antonelli M, Bruno Blasi FA, Casagranda I, Chieregato A, Fumagalli R, et al. Procalcitonin-guided antibiotic therapy: an expert consensus. Clin Chem Lab Med 2018;56:1223-9.

12. Eidizadeh A, Asif AR, von Ahsen N, Binder L, Schnelle M. Differences in procalcitonin measurements between three BRAHMSpartnered immunoassays (Liaison, Elecsys and Architect). Clin Chem Lab Med 2019;57:e207-10.

13. Brodska H, Valenta J, Pelinkova K, Stach Z, Sachl R, Balik M, et al. Diagnostic and prognostic value of presepsin vs. established biomarkers in critically ill patients with sepsis or systemic inflammatory response syndrome. Clin Chem Lab Med 2018;56:658-68.

14. Karrasch M, Geraci J, Sachse S, Rödel J, Löffler B, Bauer M, et al. Early adjustment of antimicrobial therapy after PCR/electrospray ionization mass spectrometry-based pathogen detection in critically ill patients with suspected sepsis. Clin Chem Lab Med 2018;56:e207-9.

15. Schuetz P, Beishuizen A, Broyles M, Ferrer R, Gavazzi G, Gluck $\mathrm{EH}$, et al. Procalcitonin (PCT)-guided antibiotic stewardship: an international experts consensus on optimized clinical use. Clin Chem Lab Med 2019;57:1308-18.

16. Plebani M, Laposata M, Lippi G. A manifesto for the future of laboratory medicine professionals. Clin Chim Acta 2019;489:49-52.

17. Chambliss AB, Hayden J, Colby JM. Evaluation of procalcitonin immunoassay concordance near clinical decision points. Clin Chem Lab Med 2019;57:1414-21.

Prof. Giuseppe Lippi, Section of Clinical Biochemistry, University Hospital of Verona, Piazzale LA Scuro, 37134 Verona, Italy, E-mail: giuseppe.lippi@univr.it. https://orcid.org/0000-0001-9523-9054 